Montès begins with a discussion of the various roles capitals play as administrative centers and as loci for political and judicial activity, and as "places of memory" in historical, social, and cultural contexts. Additional topics explored in turn include the geography of capital locations from colonial times to the present, an examination of the factors involved in capital choice, and comparative analyses of the demographic and economic characteristics of capital cities prior to and since 1950 . He also applies a conceptual model for the location and urban-economic development of capital cities to the cities of Columbus, Des Moines, and Frankfort, and discusses the current status of selected former capitals. The book concludes with a consideration of the role of state capitals today as "symbols of American democracy."

The narrative is well supported with factual detail. I was struck by some similarities to research by the mid-twentieth-century urban and cultural geographers Chauncy Harris and Wilbur Zelinsky, each of whom conducted research using cities or places as units of analysis, creating classifications and typologies based on socio-demographic, economic, and cultural factors. Curiously, neither of these researchers are listed in this book's index. However, quantitative analysis with mathematical models, typical of both Harris and Zelinsky, does not feature prominently in the discourse provided by Montès.

Having carefully read this monograph, I can say that I learned some things about state capitals (and capitols) that I had not previously known. However, I did not come away convinced that the subject was truly worthy of this level of scholarly investigation. The explanatory model developed in the later chapters and examined through three case studies seems contrived as well as overly complex. The vignettes of each of these capital cities, on the other hand, could stand on their own, losing little of their substance in the model's absence.

American Capitals is well illustrated, with photographs, tables, occasional diagrams, and a few maps. The end matter includes appendices with demographic and historical tables, a state-by-state chronology of colonial, territorial, and state capitals, and finally, notes, references, and an index.

In the final analysis, while I'm still not sure that the topic of state capitals in the US is worthy of a full-length book treatment, I am convinced that Christian Montès has examined them from almost every conceivable social scientific perspective, save perhaps scientific qualitative approaches with focus groups, participant observation research, or multivariable modeling. While his writing is informative and in some sections quite engaging, the reader sometimes gets lost in a mire of technical details. Given that no one had ever attempted to write a book like this previously, American Capitals is likely to be the go-to source for details about the geography of the capitals of US states for many years to come.

This monograph is a recent addition to one of the most venerable scholarly monograph series in North American academic geography. I was pleased to see this tradition from the University of Chicago continue, albeit with a lapse of eight years since the previous volume, and hope that more works are in the pipeline.

\title{
MAKING SPATIAL DECISIONS USING GIS AND LIDAR: A WORKBOOK
}

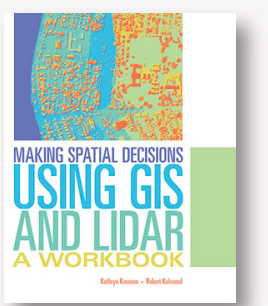

by Kathryn Keranen and Robert Kolvoord

Esri Press, 2016

216 pages. $\$ 79.99$, softcover.

ISBN: 978-1-58948-429-0

Review by: Greg March

Making Spatial Decisions Using GIS and Lidar: A Workbook, is the third book in the Making Spatial Decisions series. This tutorial is geared towards users who have basic GIS skills and want to learn how to integrate and analyze Light Detection and Ranging (lidar) data as part of a GIS project to solve problems and create high resolution maps. Below is the four-step workflow described in the book:

1. Define the problem or scenario.

2. Identify the deliverables needed to support decisions.

3. Document, set environments, and examine the data.

4. Perform analysis starting with a basemap. 
The table of contents includes ten modules, each with a unique scenario. Each module includes three projects. The first project provides the user with step-by-step instructions to help solve the main issue posed in the scenario. The second involves a similar challenge, but without stepby-step instructions: the user should rely on what they have learned in the first project to help solve the main problem in the second. The third project includes an "on your own" section with different challenges to choose from.

\section{MODULES 1-5}

The first five modules involve the situation of a fictional company asked to complete work for the cities of Baltimore, MD (Project 1) and San Francisco, CA (Project 2). Projects 1 and 2 each come with four recommended deliverables and a set of step-by-step instructions. What's nice about Project 1 is that it also includes a set of computer screen shots to help the user follow along. Project 2 does not include screenshots, in order to make things a bit more challenging. Project 3 provides a nice list of topics, from which the user can choose their own project based on their interest. These topics include forest characterization / canopy height and density, flood modeling, finding faults, geomorphic mapping, stream slope, archeology field campaigns, mining / calculation of ore volumes, and wind farm optimization.

\section{Module 1: Basic Lidar Techniques}

In this first scenario, your GIS company is tasked to check the quality of some LAS (lidar) data, conduct an analysis, edit the lidar data, and then produce both $2 \mathrm{D}$ and $3 \mathrm{D}$ surface and profile maps.

\section{Module 2: 2D and 3D Models}

Using lidar data in a GIS, your GIS company has been tasked to build raster and terrain models of the cities of Baltimore and San Francisco.

\section{Module 3: Volumetric Analysis and Shadow Maps}

The two cities have limited above-ground parking and need to explore possibilities for underground parking. Your GIS company has been tasked to determine how much land would need to be excavated where new buildings are under development. The cities also need a shadow map to guide decisions on where eating areas with benches will be constructed.

\section{Module 4: Visibility Analysis and Comparison}

Again, the two cities want to provide their residents with continuous cell phone coverage and have employed a new telecommunications company to produce a visibility analysis that includes generating a viewshed using digital surface models (DSMs) with the use of lidar data. Where problematic coverage areas occur, line-of-sight analysis is performed.

\section{Module 5: Surging Seas}

Baltimore and San Francisco want your GIS company to create new flood insurance rate maps (FIRMs) for the Federal Emergency Management Agency (FEMA) to be accessible via ArcGIS Online so that citizens can type in their address and access accurate information for their area. These new FIRMs should be constructed using the following hurricane inundation zones: 5 feet above normal, 8 feet above normal, 12 feet above normal, and $18+$ feet above normal.

Modules 1-5 all use the same data so there is only one data folder for them all, plus one results folder for each project.

\section{MODULES 6 AND 7}

These modules involve solving a set of campus-based problems centered around an alliance between James Madison University and the University of San Francisco. Both modules use the same data so there is one folder for data and one for results.

\section{Module 6: Corrected 3D Campus Modeling}

Before each campus can determine where to add solar panels on rooftops, university researchers will use features from the lidar data to reclassify (correct) lidar feature classification codes where errors had been found. Researchers will then use ArcScene to visualize the campus (in 3D) as a surface and as set of contours.

\section{Module 7: Location of Solar Panels}

Once the new lidar feature classification codes have been set, the researchers at James Madison and San Francisco will create rooftop (heat) maps to determine where solar panels will be placed for maximum operational efficiency. 


\section{MODULES 8-10}

Modules 8-10 each use different sets of data so there are separate folders for each scenario.

\section{Module 8: Shoreline Change After Hurricane Sandy}

The United States Geological Survey (USGS) has hired your GIS company to analyze changes to the New Jersey and New York shoreline after Hurricane Sandy. With the use of pre- and post-event lidar data, the USGS would like your company to produce pre- and post-event elevation maps to document elevation decline from erosion, shoreline retreat, and the deposit of soil due to wave surge.

\section{Module 9: Forest Vegetation Height}

This module involves forest vegetation height and how land managers can use lidar data to better manage forest ecosystems. Using lidar to track forest evolution, The Nature Conservancy's Virginia and Pennsylvania chapters wish to determine canopy height as a substitute for the age of the forest. This is done by subtracting the bare earth surface (DEM) from the first return surface (DSM).

\section{Module 10: Depressional Wetland Delineation from Lidar}

This last module focuses on identifying the location of depressional wetlands using lidar. The State of Florida wants to establish a wetlands center in order to perform advanced research by comparing traditional delineation methods (on-site fieldwork with a topographic map) with new methods performed off-site using lidar. The State would like a comprehensive comparative analysis of depressional wetlands that documents and compares results of the traditional methods versus the new.

\section{EVALUATION}

This workbook follows the general format of the others in the Making Spatial Decisions series, but with an improved layout, making it easier to follow than earlier ones.

In the Introduction, on page xii, there is a set of bullets that list skills the user should have (such as a basic knowledge of lidar and how it is used) prior to taking the tutorial. While there is a description of basic lidar terminology, a comprehensive glossary would have been helpful.
Downloading the instructional materials from the Esri Press "Book Resources" webpage was easy. I tested the GIS data in the scenarios using ArcGIS Desktop 10.3.1 with Windows 7 and the screenshots look the same as the ones in the book. Sometimes screenshots can look a bit different (between ArcGIS versions), which could make following the tutorial more difficult.

There are very helpful screenshots in this workbook to aid the user with completing the scenarios, but adding additional screenshots to the first few modules could better prepare the user to successfully complete all of the project scenarios within the workbook. As I followed a few of the scenarios, it wasn't always easy to get through them. Some steps didn't have enough information to guide the user, or there was information missing that could leave the user scratching their head. I have an intermediate skill level using ArcGIS, with a basic knowledge of lidar, and I had trouble understanding what was being asked at times and/ or what to do next. Some examples:

- On page 5, step 3, the user is asked to enter map document properties, but there is no mention of the Default Geodatabase field. When filling out the map document properties box, the Default Geodatabase automatically maps itself (at least on my computer). This may not happen on other computers, but a sentence or two describing this field would be helpful to the user since this is an important step in the documentation process.

- Page 10, step 13 directs the user to "Set the Baltimore_tiles dataset as the input." Should the user to go back to the Geoprocessing tab, click Environment Settings, then reset the file path in Workspace? More information is needed here.

- On page 109, step 1, the instructions read: "Add the study_area feature class and make it hollow. Add the Imagery with Labels basemap." I located the study_ area feature class file and made it hollow, but there is no mention of the basemap file in the workbook and I could not find a file with this name (Imagery with Labels) in ArcCatalog. While I did remember that ArcGIS Online has this web basemap, it would have been helpful if there was a note that the user can download the Imagery with Labels basemap from the ArcGIS website. Another, different, basemap is 
required in step 3 on page 136, and again its sourcing from ArcGIS Online is not mentioned.

Some of the instructional steps within the scenarios require the provision of more information if users are to complete each scenario. As with most GIS tutorials, individuals who take this tutorial by themselves or as a group should expect to devote a significant amount of time to working through each scenario.

This book lends itself best to a classroom setting where students can ask questions of their instructor, or fellow students who have been through the workbook scenarios. Individuals who take this tutorial solo, and without having a basic understanding of lidar and how it functions, could have some difficulty getting through it. Taking this tutorial in a classroom setting where students are divided into small groups would be to the individuals' advantage vs. taking it on their own.
It is noted in the Acknowledgements section that the workbook was created with contributions from students and teachers from the Geospatial Semester held at James Madison University, and that high school students who participated in the Geospatial Semester helped test each module in order to provide feedback.

All-in-all, this is a useful tutorial workbook for individuals who already have a basic knowledge of lidar and possess basic GIS skills. Although some of the instructional steps for following the scenarios would benefit from being a bit more detailed, the authors did well in creating real-world scenarios that represent a variety of disciplines, and the workbook enables users with GIS experience to expand their skill set (utilizing lidar) to solve problems that face the environment. I would recommend this workbook to university faculty (from select academic disciplines) with the suggestion that they integrate the workbook as part of an Introduction to Lidar course or Remote Sensing Technology course.

\section{CARTOGRAPHIC JAPAN: A HISTORY IN MAPS}

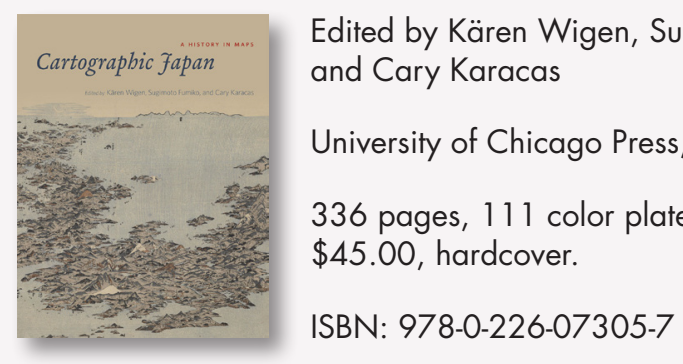

Review by: Mark Denil

For the western observer, Japan is an enigmatic land. It is both like and unlike, familiar and alien, clear and clouded, straightforward and not-quite; and it is all these things all at the same time. As Lafcadio Hearn wrote over a century ago, recounting the words of a close Japanese friend: "When you find, in four or five years more, that you cannot understand the Japanese at all, then you will begin to know something about them" (Hern 1904, 5).

Cartographic Japan: A History in Maps, from the University of Chicago Press, is expressly intended to open a window for the non-Japanese reader on a sweeping landscape of Japanese cartographic artifacts, history, and scholarship. The fifty-two short essays by forty-eight authors, "each focused on one or two maps related to the contributor's specialty" (3), are organized in four roughly chronological Parts and then further into thematic sub-groups. Although there are occasional references from one to another, for the most part each essay stands alone.

Japanese cartography, like much of Japanese culture and technology, developed in a cyclic manner. Foreign influences would, from time to time, wash over native practice; sometimes swamping local traditions, sometimes being absorbed and assimilated, and sometimes creating new streams of tradition alongside those already existing. In each case, that which remained and flourished would itself become Japanese. Cartographic Japan examines a large number of maps representing developing cartographic practices, and offers commentary on how many of these streams of infiltrations, minglings, and adaptations played out over the years.

Prevailing historical, political, and social conditions always frame the environment for cartographic practice, production, and products, and in Cartographic Japan the three most recent Japanese historical periods are used to divide the essays into four main Parts. These are titled: 